\title{
Existence of efficient envy-free allocations of a heterogeneous divisible commodity with nonadditive utilities
}

\author{
Farhad Hüsseinov • Nobusumi Sagara
}

Received: 13 September 2011 / Accepted: 23 November 2012 / Published online: 8 January 2013

(C) Springer-Verlag Berlin Heidelberg 2013

\begin{abstract}
This paper studies the existence of Pareto optimal, envy-free allocations of a heterogeneous, divisible commodity for a finite number of individuals. We model the commodity as a measurable space and make no convexity assumptions on the preferences of individuals. We show that if the utility function of each individual is uniformly continuous and strictly monotonic with respect to set inclusion, and if the partition matrix range of the utility functions is closed, a Pareto optimal envy-free partition exists. This result follows from the existence of Pareto optimal envy-free allocations in an extended version of the original allocation problem.
\end{abstract}

\section{Introduction}

Dividing scarce resources among members of a society to fulfill efficiency and fairness is a central theme in group decision-making problems. This paper studies the existence of Pareto optimal, envy-free allocations of a heterogeneous, divisible commodity for a finite number of individuals. Following the tradition of Banach-Steinhaus' cake cutting problem (see Steinhaus 1948), the commodity is modeled as a measurable space $(\Omega, \mathcal{F})$ and the preferences of each individual are defined on the $\sigma$-algebra $\mathcal{F}$ that describes feasible pieces of the heterogeneous divisible commodity $\Omega$.

An earlier version of this paper was presented at the 4th World Congress of the Game Theory Society held in Istanbul, Turkey.

\section{F. Hüsseinov}

Department of Economics, Bilkent University, 06800 Bilkent, Ankara, Turkey e-mail: farhad@bilkent.edu.tr

N. Sagara $(\bowtie)$

Faculty of Economics, Hosei University, 4342, Aihara, Machida, Tokyo194-0298, Japan

e-mail: nsagara@hosei.ac.jp 
A classical result from Dubins and Spanier (1961) demonstrates the existence of envy-free partitions under the assumption that the utility functions of each individual are nonatomic finite measures. In turn, Weller (1985) showed the existence of Pareto optimal envy-free partitions under the additional assumption that the measures that represent the utility functions are mutually, absolutely continuous. Because of the additivity of utility functions in these studies, Lyapunov's convexity theorem (see Lyapunov 1940) guarantees the compactness and convexity of the associated utility possibility set, a crucial property for the existence of various solutions. (For a comprehensive treatment of the additive utility case, see Barbanel 2005.)

In the case of nonadditive preferences on $\sigma$-algebras, the abovementioned conclusion no longer holds. This difficulty forces one to impose additional structures on the underlying measurable space $(\Omega, \mathcal{F})$. For instance, Dall' Aglio and Maccheroni (2009) assumed certain kinds of continuity and convexity axioms to show the existence of envy-free partitions of a unit simplex into polytopes.

Hüsseinov (2011) considered an alternative problem where each individual evaluates feasible pieces of $\Omega$ in terms of a finite number of subjective attributes determined by nonatomic vector measures. This enables one to identify the preferences of each individual on a subjective consumption set with one on compact and convex subset of a Euclidean space. The advantage of this approach lies in the fact that under the assumptions of the continuity, convexity, and monotonicity of preferences, the existence of competitive equilibrium partitions, core partitions, and Pareto optimal group envy-free partitions are established without the completeness and transitivity of preferences.

The purpose of this paper is to establish the existence of Pareto optimal envy-free partitions without convexity assumptions on preferences. To this end, we identify each measurable subset of $\Omega$ with its characteristic function and endow $\sigma$-algebra $\mathcal{F}$ with the topology induced by the weak* topology of $L^{\infty}(\Omega, \mathcal{F}, \mu)$, where $\mu$ is a nonatomic finite measure. We prove that if the utility function of each individual on $\mathcal{F}$ is uniformly continuous with respect to this topology, strictly monotonic with respect to set inclusion, and if the partition matrix range of the utility functions is closed, then a Pareto optimal envy-free partition exists.

Along the lines of Akin (1995), Hüsseinov and Sagara (2012) we extend the original partitioning problem with commodity space $(\Omega, \mathcal{F})$ to an allocation problem with commodity space $L^{\infty}(\Omega, \mathcal{F}, \mu)$ that one obtains upon extending the utility functions on $\mathcal{F}$ to $L^{\infty}(\Omega, \mathcal{F}, \mu)$. The existence of Pareto optimal envy-free partitions in the original problem follows from the existence of those in the extended allocation problem.

A technique in Varian (1974), developed in a framework quite different from the present analysis, proves useful in the proof of our main result. The crucial argument is as follows:

- the Pareto frontier is homeomorphic to the unit simplex (see also Mas-Colell 1986; Sagara 2008);

- for every Pareto optimal allocation, an individual exists whom no one envies;

- the intersection of a suitable closed covering of the Pareto frontier is nonempty by the Scarf version of the Knaster-Kuratowski-Mazurkiewicz (KKM) theorem (see Scarf 1967). 
In contrast to Hüsseinov (2011), our formulation employs utility functions represented by rational preferences, but the common consumption set $\mathcal{F}$ is intrinsically infinite-dimensional. As a cost of infinite-dimensionality, we must assume the closedness of the utility possibility set. While Hüsseinov (2011) provided an indirect proof of the existence of Pareto optimal envy-free partitions using the equilibrium existence theorem by Gale and Mas-Colell (1975), we present a direct proof without price systems, based on the KKM theorem, which enables us to dispense with any convexity assumption.

The outline of the paper is as follows. In Sect.2, we first review Lyapunov's convexity theorem and its variant in Dvoretsky et al. (1951). We then show that if $\mu$ is a nonatomic finite measure, then the set of characteristic functions is weakly* dense in the space $\mathcal{I}$ of measurable functions in $L^{\infty}(\Omega, \mathcal{F}, \mu)$ that have values in the closed unit interval. Uniformly continuous functions on $\mathcal{F}$ and their extensions to $\mathcal{I}$ are discussed together with the monotonicity condition. The main theorems on the existence of Pareto optimal envy-free partitions are stated in Sect. 3. There, it is shown how the original allocation problem in $(\Omega, \mathcal{F})$ can be extended to a suitable allocation problem in $L^{\infty}(\Omega, \mathcal{F}, \mu)$. Appendix includes the definitions concerning uniform spaces and the argument on the structure of the Pareto frontier.

\section{Preliminaries}

\subsection{Lyapunov's convexity theorem}

Let $(\Omega, \mathcal{F})$ be a measurable space, where $\mathcal{F}$ is a $\sigma$-algebra of subsets of a nonempty set $\Omega$. A finite signed measure $\mu$ on $(\Omega, \mathcal{F})$ is nonatomic if for every $A \in \mathcal{F}$ with $|\mu|(A)>0$ there exists $E \subset A$ such that $0<|\mu|(E)<|\mu|(A)$, where $|\mu|$ is the total variation measure of $\mu$.

Let $\mu_{1}, \ldots, \mu_{m}$ be finite signed measures on $(\Omega, \mathcal{F})$. The range of an $\mathbb{R}^{m}$-valued vector measure $\vec{\mu}=\left(\mu_{1}, \ldots, \mu_{m}\right)$ is given by:

$$
\mathcal{R}(\vec{\mu})=\left\{\vec{\mu}(A) \in \mathbb{R}^{m} \mid A \in \mathcal{F}\right\} .
$$

The integral of a measurable function $f: \Omega \rightarrow \mathbb{R}$ with respect to the signed measure $\mu_{i}$ is denoted as $\mu_{i}(f)$ and the integral with respect to the vector measure $\vec{\mu}$ is given by $\vec{\mu}(f)=\left(\mu_{1}(f), \cdots \mu_{m}(f)\right)$. For the finite measure given by $\mu=\sum_{i=1}^{m}\left|\mu_{i}\right|$, we denote by $L^{\infty}(\Omega, \mathcal{F}, \mu)$ the space (of $\mu$-equivalence classes) of $\mu$-essentially bounded functions on $\Omega$ with the $\mu$-essential sup norm.

The following result is due to Lyapunov (1940).

Lyapunov's Convexity Theorem Let $\vec{\mu}=\left(\mu_{1}, \ldots, \mu_{m}\right)$ be a vector measure such that $\mu_{1}, \ldots, \mu_{m}$ are nonatomic finite signed measures. Then $\mathcal{R}(\vec{\mu})$ is compact and convex in $\mathbb{R}^{m}$ and

$$
\mathcal{R}(\vec{\mu})=\left\{\vec{\mu}(f) \in \mathbb{R}^{m} \mid 0 \leq f \leq 1, f \in L^{\infty}(\Omega, \mathcal{F}, \mu)\right\} .
$$


A proof of Lyapunov's convexity theorem can be found in Rudin (1991, Theorem 5.5), which is based on the elegant proof by Lindenstrauss (1966).

An $n$-partition of $\Omega$ is an ordered $n$-tuple $\left(A_{1}, \ldots, A_{n}\right)$ of measurable sets whose union is $\Omega$ and which are pairwise disjoint. Let $\mathcal{P}_{n}$ be the set of $n$-partitions of $\Omega$. The partition matrix range of $\vec{\mu}$ is given as:

$$
\mathcal{M}(\vec{\mu})=\left\{\left(\mu_{i}\left(A_{j}\right)\right)_{\substack{1 \leq i \leq m \\ 1 \leq j \leq n}} \in \mathbb{R}^{m \times n} \mid\left(A_{1}, \ldots, A_{n}\right) \in \mathcal{P}_{n}\right\}
$$

where $m \times n$-matrix $\left(\mu_{i}\left(A_{j}\right)\right)$ is identified with a vector in $\mathbb{R}^{m \times n}$.

We exploit the following useful result due to Dvoretsky et al. (1951), which follows from Lyapunov's convexity theorem.

Proposition 2.1 (Dvoretsky et al.) Let $\vec{\mu}=\left(\mu_{1}, \ldots, \mu_{m}\right)$ be a finite-dimensional vector measure such that $\mu_{1}, \ldots, \mu_{m}$ are nonatomic finite signed measures. Then $\mathcal{M}(\vec{\mu})$ is a compact convex set in $\mathbb{R}^{m \times n}$ and

$$
\mathcal{M}(\vec{\mu})=\left\{\begin{array}{l|l}
\left(\mu_{i}\left(f_{j}\right)\right)_{\substack{1 \leq i \leq m \\
1 \leq j \leq n}} \in \mathbb{R}^{m \times n} \mid \begin{array}{l}
\sum_{j=1}^{n} f_{j}=1, f_{1}, \ldots, f_{n} \geq 0 \\
f_{1}, \ldots, f_{n} \in L^{\infty}(\Omega, \mathcal{F}, \mu)
\end{array}
\end{array}\right\}
$$

When $n=2$, Proposition 2.1 is reduced to Lyapunov's convexity theorem. A proof of Proposition 2.1 based on the technique of Lindenstrauss (1966), which employs the Krein-Milman theorem, is provided by Akin (1995). The extension of this result to the case $n=\infty$ is given by Edwards (1987) using the Lindenstrauss technique, while its elementary proof, which uses measure-theoretic arguments only, is given by Khan and Rath (2009).

\section{$2.2 L^{\infty}$-Spaces with nonatomic finite measures}

Let $(\Omega, \mathcal{F}, \mu)$ be a $\sigma$-finite measure space. The norm dual of $L^{1}(\Omega, \mathcal{F}, \mu)$ is $L^{\infty}(\Omega, \mathcal{F}, \mu)$ with the duality given by $\langle f, g\rangle=\int f g d \mu$ for $f \in L^{\infty}(\Omega, \mathcal{F}, \mu)$ and $g \in L^{1}(\Omega, \mathcal{F}, \mu)$. The weak ${ }^{*}$ topology of $L^{\infty}(\Omega, \mathcal{F}, \mu)$ is the topology obtained by taking as a neighborhood base of $f_{0} \in L^{\infty}(\Omega, \mathcal{F}, \mu)$ sets of the form:

$$
\mathcal{N}\left(f_{0} ; g_{1}, \ldots, g_{m}, \varepsilon\right)=\left\{\begin{array}{l|l}
f \in L^{\infty}(\Omega, \mathcal{F}, \mu) & \begin{array}{l}
\left|\left\langle f, g_{i}\right\rangle-\left\langle f_{0}, g_{i}\right\rangle\right|<\varepsilon \\
i=1, \ldots, m
\end{array}
\end{array}\right\}
$$

where $m \in \mathbb{N}, g_{1}, \ldots, g_{m} \in L^{1}(\Omega, \mathcal{F}, \mu)$ and $\varepsilon>0$. The weak* topology is the coarsest topology in which every linear functional $f \mapsto\langle f, g\rangle$ on $L^{\infty}(\Omega, \mathcal{F}, \mu)$ with $g \in L^{1}(\Omega, \mathcal{F}, \mu)$ is continuous. Thus, a net $\left\{f_{\alpha}\right\}$ in $L^{\infty}(\Omega, \mathcal{F}, \mu)$ converges to $f \in L^{\infty}(\Omega, \mathcal{F}, \mu)$ in the weak* topology if and only if $\left\langle f_{\alpha}, g\right\rangle \rightarrow\langle f, g\rangle$ for every $g \in L^{1}(\Omega, \mathcal{F}, \mu)$. The weak ${ }^{*}$ topology of $L^{\infty}(\Omega, \mathcal{F}, \mu)$ is denoted as $\sigma\left(L^{\infty}, L^{1}\right)$. 
Denote by $c a(\Omega, \mathcal{F}, \mu)$ the vector space of finite signed measures that are absolutely continuous with respect to $\mu$. By the Radon-Nikodym theorem, $L^{1}(\Omega, \mathcal{F}, \mu)$ is linearly isometric to $\operatorname{ca}(\Omega, \mathcal{F}, \mu)$. Hence, the weak* topology $\sigma\left(L^{\infty}, L^{1}\right)$ can be identified with the $\sigma\left(L^{\infty}, c a\right)$-topology, whose neighborhood base of $f_{0} \in L^{\infty}(\Omega, \mathcal{F}, \mu)$ is of the form:

$$
\mathcal{N}\left(f_{0} ; \mu_{1}, \ldots, \mu_{m}, \varepsilon\right)=\left\{\begin{array}{l|l}
f \in L^{\infty}(\Omega, \mathcal{F}, \mu) & \begin{array}{l}
\left|\mu_{i}(f)-\mu_{i}\left(f_{0}\right)\right|<\varepsilon \\
i=1, \ldots, m
\end{array}
\end{array}\right\},
$$

where $m \in \mathbb{N}, \mu_{1}, \ldots, \mu_{m} \in c a(\Omega, \mathcal{F}, \mu)$ and $\varepsilon>0$.

The following result seems well known, although it is difficult to find an available proof in the literature. For completeness of exposition, we provide the proof following the argument in Einy et al. (1999).

Proposition 2.2 A finite signed measure is nonatomic if and only if it is absolutely continuous with respect to a nonatomic measure.

Proof If $\lambda$ is a nonatomic finite signed measure, then its total variation measure $|\lambda|$ is nonatomic with respect to which $\lambda$ is absolutely continuous. Conversely, let $\mu$ be a nonatomic measure and take any $\lambda \in c a(\Omega, \mathcal{F}, \mu)$. Assume, on the contrary, that there exists an atom $A \in \mathcal{F}$ of $\lambda$. Let $0<\varepsilon<|\lambda|(A)$. Since $\lambda$ is absolutely continuous with respect to $\mu$, there is $\delta>0$ such that for every $E \in \mathcal{F}$ with $\mu(E)<\delta$, we have $|\lambda|(E)<\varepsilon$. Let $m_{0}$ be a natural number such that $m_{0} \delta>\mu(A)$. By the nonatomicity of $\mu$, there exists a partition $\left(A_{1}, \ldots, A_{m}\right)$ of $A$ such that $0<\mu\left(A_{i}\right)<\mu(A) / m_{0}<\delta$ for each $i=1, \ldots, m$. Because $A$ is an atom of $\lambda$, for each $i$ either $|\lambda|\left(A_{i}\right)=0$ or $|\lambda|\left(A_{i}\right)=|\lambda|(A)$. We thus obtain $|\lambda|(A)=|\lambda|\left(A_{i}\right)<\varepsilon$ for some $i$, which is a contradiction.

Let $\mathcal{I}=\left\{f \in L^{\infty}(\Omega, \mathcal{F}, \mu) \mid 0 \leq f \leq 1\right\}$. Then $\mathcal{I}$ is weakly* compact and each measurable set $A$ in $\mathcal{F}$ is naturally identified with its characteristic function $\chi_{A}$ in $\mathcal{I}$.

A proof of the following well known fact is found in Aumann and Shapley (1974, Proposition 22.4) or Kingman and Robertson (1968, Lemma 3).

Proposition 2.3 Let $\mu$ be a nonatomic $\sigma$-finite measure. Then, the set of characteristic functions $\left\{\chi_{A} \in L^{\infty}(\Omega, \mathcal{F}, \mu) \mid A \in \mathcal{F}\right\}$ is dense in $\mathcal{I}$ and relatively compact for the weak* topology.

\subsection{Uniform continuity}

Denote by $\left[L^{\infty}(\Omega, \mathcal{F}, \mu)\right]^{2}$ the product space $L^{\infty}(\Omega, \mathcal{F}, \mu) \times L^{\infty}(\Omega, \mathcal{F}, \mu)$ and let a pair $\left(L^{\infty}(\Omega, \mathcal{F}, \mu), \mathcal{U}\right)$ be a uniform space with the base $\mathcal{B}$ for the uniformity $\mathcal{U}$, where $\mathcal{B}$ is the family of all sets $U$ of the form:

$$
U=\left\{(f, g) \in\left[L^{\infty}(\Omega, \mathcal{F}, \mu)\right]^{2}|| \mu_{i}(f)-\mu_{i}(g) \mid<\varepsilon, i=1, \ldots, m\right\},
$$

where $m \in \mathbb{N}, \mu_{1}, \ldots, \mu_{m} \in c a(\Omega, \mathcal{F}, \mu)$ and $\varepsilon>0$. Given a subset $X$ of $L^{\infty}(\Omega, \mathcal{F}, \mu)$, denote by $\mathcal{U}_{X}=\left\{U \cap(X \times X) \subset\left[L^{\infty}(\Omega, \mathcal{F}, \mu)\right]^{2} \mid U \in \mathcal{U}\right\}$ the 
relative uniformity for $X$. (For the definitions of uniformities, bases, and relative uniformities, see Appendix 5.1.)

Definition 2.1 A function $v: X \rightarrow \mathbb{R}$ is uniformly continuous if for every $\varepsilon>0$ there exists $U \in \mathcal{U}_{X}$ such that $(f, g) \in U$ implies $|v(f)-v(g)|<\varepsilon$.

Notice that the uniform and the weak* topologies are the same on $L^{\infty}(\Omega, \mathcal{F}, \mu)$. Hence, topologies induced on $X$ by these two topologies are the same (see Appendix 5.1). Our focus here is on the uniformly continuous functions defined on $X=\mathcal{F}$ and $X=\mathcal{I}$.

A set function $v: \mathcal{F} \rightarrow \mathbb{R}$ is uniformly continuous if and only if for every $\varepsilon>0$ there exist $\mu_{1}, \ldots, \mu_{m} \in c a(\Omega, \mathcal{F}, \mu)$ and $\delta>0$ such that for every $A, B \in \mathcal{F}$ satisfying $\left|\mu_{i}(A)-\mu_{i}(B)\right|<\delta$ for $i=1, \ldots, m$, we have $|v(A)-v(B)|<\varepsilon$. A function $\hat{v}: \mathcal{I} \rightarrow \mathbb{R}$ is uniformly continuous if and only if for every $\varepsilon>0$ there exist $\mu_{1}, \ldots, \mu_{m} \in c a(\Omega, \mathcal{F}, \mu)$ and $\delta>0$ such that for every $f, g \in \mathcal{I}$ satisfying $\left|\mu_{i}(f)-\mu_{i}(g)\right|<\delta$ for $i=1, \ldots, m$, we have $|\hat{v}(f)-\hat{v}(g)|<\varepsilon$.

Theorem 2.1 A uniformly continuous set function on $\mathcal{F}$ has a unique uniformly continuous extension to $\mathcal{I}$.

Proof As $\mathcal{F}$ is dense and its weak* closure coincides with the weakly* compact set $\mathcal{I}$ by Proposition 2.3, it is also dense and compact in the uniform topology $\tau_{\mathcal{U}}$. Therefore, the uniform continuity on $\mathcal{F}$ implies the existence of a unique uniformly continuous extension to $\mathcal{I}$ by Theorem 5.1 in Appendix 5.1.

Example 2.1 Let $\vec{\mu}=\left(\mu_{1}, \ldots, \mu_{m}\right)$ be an $m$-dimensional vector measure whose components are nonatomic finite measures of $(\Omega, \mathcal{F})$. Define the nonatomic finite measure $\mu$ by $\mu=\sum_{i=1}^{m} \mu_{i}$ and consider $L^{\infty}(\Omega, \mathcal{F}, \mu)$. Then, we have $\mu_{1}, \ldots, \mu_{m} \in$ $c a(\Omega, \mathcal{F}, \mu)$. Let $\varphi: \mathcal{R}(\vec{\mu}) \rightarrow \mathbb{R}$ be a continuous function and define the set function $v: \mathcal{F} \rightarrow \mathbb{R}$ by $v=\varphi \circ \vec{\mu}$. Then, $v$ is uniformly continuous and its uniformly continuous extension $\hat{v}: \mathcal{I} \rightarrow \mathbb{R}$ is given by $\hat{v}(f)=\varphi(\vec{\mu}(f))$ for $f \in \mathcal{I}$. The set functions and their uniformly continuous extensions of this type are investigated at length in Hüsseinov and Sagara (2012) to demonstrate the existence of fuzzy core allocations in an exchange economy with a heterogeneous divisible commodity.

The uniform continuity of set functions on $\mathcal{F}$ is stronger than the continuity of those at every set $A \in \mathcal{F}$ in the measure-theoretic sense: $v$ is continuous at $A \in \mathcal{F}$ if for every sequence $\left\{A^{k}\right\}$ in $\mathcal{F}$ with $A^{k} \uparrow A$ and every sequence $\left\{A^{k}\right\}$ in $\mathcal{F}$ with $A^{k} \downarrow A$, we have $\lim _{k} v\left(A^{k}\right)=v(A)$. It is also stronger than the $\vec{\mu}$-uniform continuity introduced by Hüsseinov and Sagara (2012): $v$ is $\vec{\mu}$-uniform continuous if for every $\varepsilon>0$ there is $\delta>0$ such that $\|\vec{\mu}(A \triangle B)\|<\delta$ implies $|v(A)-v(B)|<\varepsilon$, where $\vec{\mu}$ is a finite-dimensional vector measure whose component measures are in $c a(\Omega, \mathcal{F}, \mu) A \triangle B \in \mathcal{F}$ is the symmetric difference of $A$ and $B$ in $\mathcal{F}$, and $\|\cdot\|$ is the Euclidean norm.

One of the merits of such a stronger notion of continuity lies in the fact that uniformly continuous set functions on $\mathcal{F}$ possess a weakly* continuous extension to $\mathcal{I}$, as shown in Theorem 2.2. The notions of uniform continuity and uniformly continuous extensions introduced in this paper originated in Aumann and Shapley (1974, p. 147), who formulated it on the space of bounded measurable functions with values in the 
closed unit interval, endowed with the "NA-topology". A variant of these notions is found in Einy et al. (1999). We develop here a systematic treatment of uniform continuity in terms of the uniform and weak* topologies on $L^{\infty}(\Omega, \mathcal{F}, \mu)$, which conforms with the standard definition of uniform spaces. (See Kelley 1955, Chapter 6).

\section{$2.4 \mu$-Monotonicity}

Definition 2.2 (i) A set function $v: \mathcal{F} \rightarrow \mathbb{R}$ is $\mu$-monotone if there exists a constant $k \geq 0$ such that $v(A)+k \mu(B \backslash A) \leq v(B)$ whenever $A \subset B$. When $k>0$, we say that $\nu$ is strictly $\mu$-monotone.

(ii) A function $\hat{v}: \mathcal{I} \rightarrow \mathbb{R}$ is $\mu$-monotone if there exists a constant $k \geq 0$ such that $\hat{v}(f)+k \mu(g-f) \leq \hat{v}(g)$ whenever $f \leq g$. When $k>0$, we say that $\hat{v}$ is strictly $\mu$-monotone.

The intended meaning of this definition is that "the marginal utility" $(v(B)-$ $\nu(A)) / \mu(B \backslash A)$ of the added piece $B \backslash A$ in terms of measure $\mu$ has a uniform lower bound $k$. Note that when $k=0$, the above definitions reduce to the monotonicity of $v$ and $\hat{v}$ in the sense that $A \subset B$ implies $v(A) \leq v(B)$ and $f \leq g$ implies $\hat{v}(f) \leq \hat{v}(g)$. The strict $\mu$-monotonicity of set functions on $\mathcal{F}$ is somewhat stronger than the strict monotonicity condition introduced by Sagara (2008): $v(A)<v(B)$ whenever $A \subset B$ and $\mu(A)<\mu(B)$.

The next result guarantees that uniformly continuous set functions on $\mathcal{F}$ with (strict) $\mu$-monotonicity have uniformly continuous extensions to $\mathcal{I}$ with the same property.

Theorem 2.2 Let $\mu$ be a nonatomic finite measure. Then, a uniformly continuous set function on $\mathcal{F}$ is (strictly) $\mu$-monotone if and only if it has a uniformly continuous, (strictly) $\mu$-monotone extension to $\mathcal{I}$.

Proof If $\hat{v}: \mathcal{I} \rightarrow \mathbb{R}$ is a (strictly) $\mu$-monotone extension of a uniformly continuous set function $v: \mathcal{F} \rightarrow \mathbb{R}$, then obviously $v$ is (strictly) $\mu$-monotone. Suppose, conversely, that $v$ is uniformly continuous and (strictly) $\mu$-monotone. By Theorem 2.1, $v$ has a uniformly continuous extension $\hat{v}: \mathcal{I} \rightarrow \mathbb{R}$. Let $f, g \in \mathcal{I}$ and $f \leq g$. By the uniform continuity of $\hat{v}$, for every $\varepsilon>0$, there exist $\mu_{1}, \ldots, \mu_{m} \in c a(\Omega, \mathcal{F}, \mu)$, and $\delta>0$ such that for every $f^{\prime}, g^{\prime} \in \mathcal{I}$ satisfying $\left|\mu_{i}\left(f^{\prime}\right)-\mu_{i}(f)\right|<\delta$ and $\left|\mu_{i}\left(g^{\prime}\right)-\mu_{i}(g)\right|<\delta$ for $i=1, \ldots, m$, we have $\left|\hat{v}\left(f^{\prime}\right)-\hat{v}(f)\right|<\varepsilon$ and $\left|\hat{v}\left(g^{\prime}\right)-\hat{v}(g)\right|<\varepsilon$. Proposition 2.2 guarantees that $\mu_{1}, \ldots, \mu_{m}$ are nonatomic finite signed measures. Define the $(m+1)$-dimensional vector measure by $\vec{\lambda}=\left(\mu_{1}, \ldots, \mu_{m}, \mu\right)$. Then, by Lyapunov's convexity theorem there exist $A, B \in \mathcal{F}$ with $A \subset B$ such that $\vec{\lambda}(A)=\vec{\lambda}(f)$ and $\vec{\lambda}(B)=\vec{\lambda}(g)$. Setting $f^{\prime}=\chi_{A}$ and $g^{\prime}=\chi_{B}$ yields $|\nu(A)-\hat{v}(f)|<\varepsilon$ and $|v(B)-\hat{v}(g)|<\varepsilon$. As $v(A) \leq v(B)-k \mu(B \backslash A)$ by the (strict) $\mu$-monotonicity of $\nu$, we have $\hat{v}(f)-\varepsilon<\hat{v}(g)-k \mu(B \backslash A)+\varepsilon=\hat{v}(g)-k \mu(g-f)+\varepsilon$. In view of the arbitrariness of $\varepsilon$, we obtain $\hat{v}(f) \leq \hat{v}(g)-k \mu(g-f)$. Hence, $\hat{v}$ is (strictly) $\mu$-monotone.

Example 2.2 Let $v=\varphi \circ \vec{\mu}$ be the set function studied in Example 2.1. If $\varphi$ is continuous and monotone, then by Theorem 2.2, $v$ has a uniformly continuous monotone extension $\hat{v}=\varphi \circ \vec{\mu}$ to $\mathcal{I}$. Assume further that $\varphi$ is strictly monotone and continuously 
differentiable on $\mathcal{R}(\vec{\mu})$. Let $k=\min \left\{\frac{\partial \varphi}{\partial x_{i}}(x) \mid x \in \mathcal{R}(\vec{\mu}), i=1, \ldots, m\right\}$. Take any $x, y \in \mathcal{R}(\vec{\mu})$ with $x \leq y$. By the mean value theorem, we have $\varphi(x)-\varphi(y)=$ $\sum_{i=1}^{m} \frac{\partial \varphi}{\partial x_{i}}\left(x^{0}\right)\left(x_{i}-y_{i}\right) \leq-k \sum_{i=1}^{m}\left(y_{i}-x_{i}\right)$, where $k>0$ by the strict monotonicity of $\varphi$, and $x^{0} \in \mathcal{R}(\vec{\mu})$ is a convex combination of $x$ and $y$. Hence, if $A \subset B$, then putting $x=\vec{\mu}(A)$ and $y=\vec{\mu}(B)$ yields $\varphi(\vec{\mu}(A)) \leq \varphi(\vec{\mu}(B))-k(\mu(B \backslash A))$. This shows that $v=\varphi \circ \vec{\mu}$ is a strictly $\mu$-monotone set function on $\mathcal{F}$ with a strictly $\mu$-monotone extension $\hat{v}=\varphi \circ \vec{\mu}$ to $\mathcal{I}$.

Given a set function $v: \mathcal{F} \rightarrow \mathbb{R}$, a set $N \in \mathcal{F}$ is $v$-null if $v(A \cup N)=v(A)$ for every $A \in \mathcal{F}$. A set function $v$ is nonatomic if, for every $v$-nonnull set $A \in \mathcal{F}$, there exists a subset $B$ of $A$ in $\mathcal{F}$ such that both $A \backslash B$ and $B$ are $\nu$-nonnull.

Example 2.3 Note that $\mu$-monotone set functions are not necessarily nonatomic even if $\mu$ is nonatomic. To illustrate this, let $\mu$ be the Lebesgue measure on the $\sigma$-hspace0ptalgebra of Borel subsets in $[0,1]$, and let $v: \mathcal{F} \rightarrow \mathbb{R}$ be defined as:

$$
v(A)= \begin{cases}\mu(A) & \text { if } 1 \notin A, \\ \mu(A)+1 & \text { otherwise }\end{cases}
$$

It is easily checked that $v$ is strictly $\mu$-monotone (with $k=1$ ), but atomic (the singleton $\{1\}$ is an atom of $v)$.

\section{Pareto optimal envy-free partitions}

\subsection{Allocations in $L^{\infty}$-spaces}

The problem of dividing a heterogeneous commodity among a finite number of individuals is formulated as the partitioning of a measurable space $(\Omega, \mathcal{F})$. Here, the set $\Omega$ is a heterogeneous divisible commodity and $\sigma$-algebra $\mathcal{F}$ of subsets of $\Omega$ describes the collection of possible pieces of $\Omega$.

There are $n$ individuals, indexed by $i=1, \ldots, n$, whose preferences on $\mathcal{F}$ are given by utility functions $v_{i}: \mathcal{F} \rightarrow \mathbb{R}, i=1, \ldots, n$. A partition $\left(A_{1}, \ldots, A_{n}\right)$ of $\Omega$ is interpreted as an allocation that gives piece $A_{i} \in \mathcal{F}$ to individual $i$. An allocation problem $\mathcal{E}=\left\langle(\Omega, \mathcal{F}),\left(v_{i}\right)_{i=1}^{n}\right\rangle$ under study is the primitive consisting of a common consumption set $\mathcal{F}$ with a measurable space $(\Omega, \mathcal{F})$ and the individuals' profile of utility functions $v_{i}$ on $\mathcal{F}$.

Definition 3.1 A partition $\left(A_{1}, \ldots, A_{n}\right)$ is:

(i) Envy free if $v_{i}\left(A_{j}\right) \leq v_{i}\left(A_{i}\right)$ for each $i, j=1, \ldots, n$.

(ii) Pareto optimal if no partition $\left(B_{1}, \ldots, B_{n}\right)$ exists such that $v_{i}\left(A_{i}\right) \leq v_{i}\left(B_{i}\right)$ for each $i=1, \ldots, n$ and $v_{j}\left(A_{j}\right)<v_{j}\left(B_{j}\right)$ for some $j$.

Let $\mu_{1}, \ldots, \mu_{n}$ be mutually absolutely continuous, finite measures. Define $\mu=$ $\sum_{i=1}^{n} \mu_{i}$. As $\mu_{i}(A)=0$ for each $i$ if and only if $\mu(A)=0$, the mutual absolute continuity of $\mu_{i}$ implies that $L^{\infty}\left(\Omega, \mathcal{F}, \mu_{i}\right)=L^{\infty}(\Omega, \mathcal{F}, \mu)$ for each $i$ because their essential supremum norms coincide. Since sets in $\mathcal{F}$ are naturally identified with 
elements with their characteristic functions, the commodity space $\mathcal{F}$ will be treated as a subset of $L^{\infty}(\Omega, \mathcal{F}, \mu)$.

An extended allocation problem $\widehat{\mathcal{E}}=\left\langle\mathcal{I},\left(\hat{v}_{i}\right)_{i=1}^{n}\right\rangle$ is the primitive consisting of a common consumption set $\mathcal{I}=\left\{f \in L^{\infty}(\Omega, \mathcal{F}, \mu) \mid 0 \leq f \leq 1\right\}$ and the individuals' profile of utility functions $\hat{v}_{i}: \mathcal{I} \rightarrow \mathbb{R}$, where $\hat{v}_{i}$ is an extension of $v_{i}$ to $\mathcal{I}$ for each $i$. Thus, $\widehat{\mathcal{E}}$ is an extension of the original allocation problem $\mathcal{E}=\left\langle(\Omega, \mathcal{F}),\left(v_{i}\right)_{i=1}^{n}\right\rangle$.

An $n$-tuple $\left(f_{1}, \ldots, f_{n}\right)$ of elements in $L^{\infty}(\Omega, \mathcal{F}, \mu)$ is an allocation of $\Omega$ if $\sum_{i=1}^{n} f_{i}=1$ and $f_{i} \in \mathcal{I}$ for each $i$. Note that $\left(A_{1}, \ldots, A_{n}\right)$ is a partition of $\Omega$ up to $\mu$-equivalence if and only if $\sum_{i=1}^{n} \chi_{A_{i}}=1$. We denote by $\mathcal{A}$ the set of allocations of $\Omega$.

Definition 3.2 An allocation $\left(f_{1}, \ldots, f_{n}\right)$ is:

(i) Envy free if $\hat{v}_{i}\left(f_{j}\right) \leq \hat{v}_{i}\left(f_{i}\right)$ for each $i, j=1, \ldots, n$.

(ii) Weakly Pareto optimal if there exists no allocation $\left(g_{1}, \ldots, g_{n}\right)$ such that $\hat{v}_{i}\left(f_{i}\right)<$ $\hat{v}_{i}\left(g_{i}\right)$ for each $i=1, \ldots, n$.

(iii) Pareto optimal if no allocation $\left(g_{1}, \ldots, g_{n}\right)$ exists such that $\hat{v}_{i}\left(f_{i}\right) \leq \hat{v}_{i}\left(g_{i}\right)$ for each $i=1, \ldots, n$ and $\hat{v}_{j}\left(f_{j}\right)<\hat{v}_{j}\left(g_{j}\right)$ for some $j$.

The existence of Pareto optimal envy-free allocations is a key step to proving that of Pareto optimal envy-free partitions.

Theorem 3.1 Let $\mu_{1}, \ldots, \mu_{n}$ be mutually absolutely continuous, nonatomic, finite measures. If for an allocation problem $\mathcal{E}=\left\langle(\Omega, \mathcal{F}),\left(v_{i}\right)_{i=1}^{n}\right\rangle, v_{i}$ is uniformly continuous and strictly $\mu_{i}$-monotone for each $i=1, \ldots, n$, then there exists a Pareto optimal envy-free allocation for $\widehat{\mathcal{E}}=\left\langle\mathcal{I},\left(\hat{v}_{i}\right)_{i=1}^{n}\right\rangle$.

To present the proof of Theorem 3.1, we use the following version of the KKM theorem from Scarf (1967) (see also Scarf 1973, Theorem 3.3.1).

Proposition 3.1 Let $\Delta_{i}=\left\{\left(x_{1}, \ldots, x_{n}\right) \in \Delta^{n-1} \mid x_{i}=0\right\}$ for each $i=1, \ldots, n$. If the collection $\left\{C_{1}, \ldots, C_{n}\right\}$ is a closed covering of $\Delta^{n-1}$ satisfying $\Delta_{i} \subset C_{i}$ for each $i$, then $\bigcap_{i=1}^{n} C_{i} \neq \emptyset$.

Proof of Theorem 3.1 Let $\mathcal{A}^{\mathrm{P}}$ be the set of all Pareto optimal allocations in $\widehat{\mathcal{E}}$. Define the set of all Pareto optimal allocations such that no one envies individual $j$ by:

$$
\mathcal{A}_{j}^{\mathrm{P}}=\left\{\left(f_{1}, \ldots, f_{n}\right) \in \mathcal{A}^{\mathrm{P}} \mid \hat{v}_{i}\left(f_{j}\right) \leq \hat{v}_{i}\left(f_{i}\right), i=1, \ldots, n\right\}
$$

Because, by the strict $\mu_{i}$-monotonicity of $\hat{v}_{i}$, there exists a Pareto optimal allocation $\left(f_{1}, \ldots, f_{n}\right)$ such that $f_{j}=0$, it is obvious that $\mathcal{A}_{j}^{\mathrm{P}}$ is nonempty for each $j=1, \ldots, n$. Thus, allocations in $\bigcap_{j=1}^{n} \mathcal{A}_{j}^{\mathrm{P}}$ are Pareto optimal and envy free. We wish to show that $\bigcap_{j=1}^{n} \mathcal{A}_{j}^{\mathrm{P}}$ is indeed nonempty.

As $\hat{v}_{i}$ is strictly $\mu_{i}$-monotone by Theorem 2.2, one may assume, without loss of generality, that $\hat{v}_{i}(0)=0$ and $\hat{v}_{i}(f) \geq 0$ for every $f \in \mathcal{I}$. Define the utility possibility set $\Gamma$ by:

$$
\Gamma=\left\{\left(x_{1}, \ldots, x_{n}\right) \in \mathbb{R}^{n} \mid \exists\left(f_{1}, \ldots, f_{n}\right) \in \mathcal{A}: 0 \leq x_{i} \leq \hat{v}_{i}\left(f_{i}\right), i=1, \ldots, n\right\}
$$


and the (weak) Pareto frontier $\Gamma^{\mathrm{P}}$ of $\Gamma$ by:

$$
\Gamma^{\mathrm{P}}=\left\{\left(x_{1}, \ldots, x_{n}\right) \in \Gamma \mid \nexists\left(y_{1}, \ldots, y_{n}\right) \in \Gamma: x_{i}<y_{i}, i=1, \ldots, n\right\} .
$$

Define also the subsets $\Gamma_{j}^{\mathrm{P}}$ and $\Gamma_{j}$ of $\Gamma$ by:

$$
\begin{aligned}
& \Gamma_{j}^{\mathrm{P}}=\left\{\left(\hat{v}_{1}\left(f_{1}\right), \ldots, \hat{v}_{n}\left(f_{n}\right)\right) \in \mathbb{R}^{n} \mid\left(f_{1}, \ldots, f_{n}\right) \in \mathcal{A}_{j}^{\mathrm{P}}\right\}, \\
& \Gamma_{j}=\left\{\left(x_{1}, \ldots, x_{n}\right) \in \Gamma^{\mathrm{P}} \mid x_{j}=0\right\} .
\end{aligned}
$$

It is easy to verify that $\Gamma_{j}$ is nonempty. As $\bigcup_{j=1}^{n} \mathcal{A}_{j}^{\mathrm{P}}=\mathcal{A}^{\mathrm{P}}$ by Proposition 5.1, we have $\bigcup_{j=1}^{n} \Gamma_{j}^{\mathrm{P}}=\Gamma^{\mathrm{P}}$. Let $h: \Delta^{n-1} \rightarrow \Gamma^{\mathrm{P}}$ be the homeomorphism established in Proposition 5.2. Then, the collection $\left\{C_{1}, \ldots, C_{n}\right\}$, where $C_{j}=h^{-1}\left(\Gamma_{j}^{\mathrm{P}}\right)$, is a closed covering of $\Delta^{n-1}$. Because $h$ gives rise to the relation

$$
\Gamma^{\mathrm{P}} \ni\left(y_{1}, \ldots, y_{n}\right)=h(x)=\rho(x) x \mapsto x \in \Delta^{n-1},
$$

in view of Proposition 5.2, we have $h^{-1}\left(\Gamma_{j}\right)=\Delta_{j}$ for each $j$. Therefore by Proposition 3.1 , there exists $x \in \bigcap_{j=1}^{n} C_{j}=h^{-1}\left(\bigcap_{j=1}^{n} \Gamma_{j}^{\mathrm{P}}\right)$. Then, for some $\left(f_{1}^{*}, \ldots, f_{n}^{*}\right) \in \mathcal{A}^{\mathrm{P}}$, we have $\left(\hat{v}_{1}\left(f_{1}^{*}\right), \ldots, \hat{v}_{n}\left(f_{n}^{*}\right)\right)=h(x) \in \bigcap_{j=1}^{n} \Gamma_{j}^{\mathrm{P}}$. Suppose that $\left(f_{1}^{*}, \ldots, f_{n}^{*}\right) \notin \mathcal{A}_{j}^{\mathrm{P}}$ for some $j$. Then, we have $\left(\hat{v}_{1}\left(f_{1}^{*}\right), \ldots, \hat{v}_{n}\left(f_{n}^{*}\right)\right) \notin \Gamma_{j}^{\mathrm{P}}$, a contradiction. Therefore, $\left(f_{1}^{*}, \ldots, f_{n}^{*}\right) \in \bigcap_{j=1}^{n} \mathcal{A}_{j}^{\mathrm{P}}$.

\subsection{Partitioning of a measurable space}

The next condition is a departure from the hypotheses required in the literature on fair division theory with additive utilities along the lines of Dubins and Spanier (1961) and Weller (1985).

Definition 3.3 A vector $\vec{v}=\left(v_{1}, \ldots, v_{n}\right)$ of individual utility functions satisfies the closedness condition if the partition matrix range:

$$
\mathcal{M}(\vec{v})=\left\{\left(v_{i}\left(A_{j}\right)\right)_{1 \leq i, j \leq n} \in \mathbb{R}^{n \times n} \mid\left(A_{1}, \ldots, A_{n}\right) \in \mathcal{P}_{n}\right\}
$$

is closed in $\mathbb{R}^{n \times n}$.

Lemma 3.1 Let $\mathcal{A}^{0}$ be the set of $\mu$-equivalence classes of partitions, that is:

$$
\mathcal{A}^{0}=\left\{\left(\chi_{A_{1}}, \ldots, \chi_{A_{n}}\right) \in\left[L^{\infty}(\Omega, \mathcal{F}, \mu)\right]^{n} \mid \sum_{i=1}^{n} \chi_{A_{i}}=1\right\} .
$$

Then, $\mathcal{A}^{0}$ is dense in $\mathcal{A}$ in the weak* topology. 
Proof Take any $\varepsilon>0$ and $\left(f_{1}, \ldots, f_{n}\right) \in \mathcal{A}$. Let $\vec{\lambda}^{1}, \ldots, \vec{\lambda}^{n}$ be finite-dimensional vector measures whose component measures are in $\operatorname{ca}(\Omega, \mathcal{F}, \mu)$. Define the finitedimensional vector measure by $\vec{\lambda}=\left(\vec{\lambda}^{1}, \ldots, \vec{\lambda}^{n}\right)$ and let $m$ be the dimension of $\vec{\lambda}$. Proposition 2.1 guarantees that there exists a partition $\left(A_{1}, \ldots, A_{n}\right)$ of $\Omega$ such that $\lambda_{k}\left(A_{i}\right)=\lambda_{k}\left(f_{i}\right)$ for each $k=1, \ldots, m$ and $i=1, \ldots, n$, where $\lambda_{k}$ is the $k$-th component of $\vec{\lambda}$. Thus, $\chi_{A_{i}} \in \mathcal{N}\left(f_{i} ; \vec{\lambda}^{i}, \varepsilon\right)$ for each $i=1, \ldots, n$. Because a neighborhood base $\mathcal{N}\left(f_{1} ; \vec{\lambda}^{1}, \varepsilon\right) \times \cdots \times \mathcal{N}\left(f_{n} ; \vec{\lambda}^{n}, \varepsilon\right)$ of $\left(f_{1}, \ldots, f_{n}\right)$ contains a characteristic function $\left(\chi_{A_{1}}, \ldots, \chi_{A_{n}}\right)$, we conclude that $\mathcal{A}^{0}$ is dense in $\mathcal{A}$ in the product weak* topology.

The central result of this paper is the following existence theorem of efficient envyfree partitions, which demonstrates the compatibility of Pareto optimality and envy freeness.

Theorem 3.2 Let $\mu_{1}, \ldots, \mu_{n}$ be mutually absolutely continuous, nonatomic, finite measures. If for an allocation problem $\mathcal{E}=\left\langle(\Omega, \mathcal{F}),\left(v_{i}\right)_{i=1}^{n}\right\rangle, v_{i}$ is uniformly continuous, strictly $\mu_{i}$-monotone for each $i=1, \ldots, n$ and the closedness condition is satisfied, then there exists a Pareto optimal envy-free partition.

Proof Let $\left(f_{1}, \ldots, f_{n}\right)$ be a Pareto optimal envy-free allocation for $\widehat{\mathcal{E}}$, established in Theorem 3.1. As it follows from the weak* continuity of $\hat{v}_{i}$ by Theorem 2.2, the closedness condition and Lemma 3.1 that:

$$
\mathcal{M}(\vec{v})=\left\{\left(\hat{v}_{i}\left(f_{j}\right)\right)_{1 \leq i, j \leq n} \in \mathbb{R}^{n \times n} \mid\left(f_{1}, \ldots, f_{n}\right) \in \mathcal{A}\right\},
$$

there exists a partition $\left(A_{1}, \ldots, A_{n}\right)$ such that $\hat{v}_{i}\left(f_{j}\right)=v_{i}\left(A_{j}\right)$ for each $i, j=$ $1, \ldots, n$. It is evident that $\left(A_{1}, \ldots, A_{n}\right)$ is a Pareto optimal envy-free partition for $\mathcal{E}$.

When each $v_{i}$ is equal to $\mu_{i}$ in Theorem 3.2, the uniform continuity, the strict $\mu_{i}$-monotonicity and the closedness condition are automatically satisfied. Hence, the Weller's result (see Weller 1985) follows from our theorem as a special case. The significance of the closedness condition on the utility possibility set for the existence of equilibria in an exchange economy with an infinite-dimensional commodity space with topological vector lattices was pointed out by Mas-Colell (1986). Likewise, in the allocation problem $\mathcal{E}=\left\langle(\Omega, \mathcal{F}),\left(\nu_{i}\right)_{i=1}^{n}\right\rangle$, the closedness of the utility possibility set (the partition range of $\vec{v}$ ):

$$
\mathcal{P}(\vec{v})=\left\{\left(v_{1}\left(A_{1}\right), \ldots, v_{n}\left(A_{n}\right)\right) \in \mathbb{R}^{n} \mid\left(A_{1}, \ldots, A_{n}\right) \in \mathcal{P}_{n}\right\}
$$

plays a crucial role for the existence of fair partitions with nonadditive utilities. This is emphasized by Sagara (2008); Sagara and Vlach (2010, 2011). For the existence of efficient envy-free partitions in Theorem 3.2, we require the closedness of $\mathcal{M}(\vec{v})$ instead of the closedness of $\mathcal{P}(\vec{v})$, which is automatically satisfied by Proposition 2.1 , whenever each utility $v_{i}$ is a nonatomic finite measure. 
Although no closedness condition is explicitly imposed in this theorem, the closedness condition on the allocation range of $\overrightarrow{\hat{v}}=\left(\hat{v}_{1}, \ldots, \hat{v}_{n}\right)$ for $\widehat{\mathcal{E}}$ :

$$
\mathcal{A}(\overrightarrow{\hat{v}})=\left\{\left(\hat{v}_{i}\left(f_{j}\right)\right)_{1 \leq i, j \leq n} \in \mathbb{R}^{n \times n} \mid\left(f_{1}, \ldots, f_{n}\right) \in \mathcal{A}\right\},
$$

is compact in view of the weak* compactness of $\mathcal{A}$ (see Lemma 3.1) and the uniform continuity of each $\hat{v}_{i}$ (see Theorem 2.1).

We stress that Theorem 3.2 requires no convexity assumption, as contrasted to Hüsseinov (2011), who showed the existence of Pareto optimal, group envy-free partitions under convexity assumptions in a different setting. While we employ the uniformly continuous utility functions on $\mathcal{F}$, Hüsseinov (2011) dispenses with the use of utility functions and deals with incomplete, nontransitive preferences on $\mathcal{F}$ induced by individual preferences on a finite-dimensional consumption set determined by a finite number of subjective attributes of elements $\mathcal{F}$ given by a finite-dimensional nonatomic vector measure. Here, such a restriction is not imposed.

Example 3.1 Consider a $2 \times 2$ exchange economy given in Berliant et al. (1992). Let $\mu$ be the Lebesgue measure on closed interval $\Omega=[0,2]$ and decompose $\Omega$ into two subintervals $\Omega_{1}=[0,1]$ and $\Omega_{2}=(1,2]$. Define the measures $\mu_{1}$ and $\mu_{2}$ by $\mu_{1}(A)=\mu\left(A \cap \Omega_{1}\right)$ and $\mu_{2}(A)=\mu\left(A \cap \Omega_{2}\right)$ for $A \in \mathcal{F}$ respectively. The utility functions of individuals are given by $\nu_{1}(A)=\varphi_{1}\left(\mu_{1}(A), \mu_{2}(A)\right)$ and $\nu_{2}(A)=\varphi_{2}\left(\mu_{1}(A), \mu_{2}(A)\right)$, where $\varphi_{i}(i=1,2)$ is a real-valued function defined on $[0,1] \times[0,1]$. Each piece $A \in \mathcal{F}$ is characterized by the two "cardinal" attributes evaluated by the two-dimensional vector measure $\left(\mu_{1}, \mu_{2}\right)$. This economy is analogous to an Edgeworth box economy.

Suppose that each $\varphi_{i}$ is a strictly increasing, continuously differentiable, concave function. Since $\mu=\mu_{1}+\mu_{2}$, it follows from Example 2.1 that each $v_{i}$ is uniformly continuous. The strict $\mu$-monotonicity of $\varphi_{i}$ is a consequence of Example 2.2. The closedness condition is satisfied by Lyapunov's convexity theorem and the continuity of $\varphi_{i}$. Therefore, weak Pareto optimal partitions coincide with Pareto optimal partitions and there exists a Pareto optimal envy-free partition in the Edgeworth box economy.

\section{Concluding remarks}

The proof of Theorem 3.1 suggests a method for detecting envy-free allocations (partitions) from the set of Pareto optimal allocations (partitions) in view of the observation that every allocation in $\bigcap_{j=1}^{n} \mathcal{A}_{j}$ is Pareto optimal and envy free. This means that one can obtain Pareto optimal envy-free allocations whenever, for each individual $j$, one can determine every Pareto optimal allocation where no individual envies $j$. Such a computational aspect might be useful for constructing a protocol for obtaining Pareto optimal envy-free allocations, especially in the case where individuals' utilities are represented by a nonatomic probability measure. This is an interesting issue that requires further research.

Acknowledgements This paper was written while Hüsseinov was visiting Hosei University. The authors benefitted from discussions with M. Ali Khan and Akira Yamazaki. Several suggestions by Ozgur Evren, 
two anonymous referees and the editor of this journal were also helpful. This research is supported by a Grant-in-Aid for Scientific Research (No.23530230) from the Ministry of Education, Culture, Sports, Science and Technology, Japan.

\section{Appendix}

\subsection{Uniform topologies on $L^{\infty}$}

A binary relation $U$ on $L^{\infty}(\Omega, \mathcal{F}, \mu)$ is a subset of $\left[L^{\infty}(\Omega, \mathcal{F}, \mu)\right]^{2}$. Its composition with itself, $U \circ U$, is defined by:

$$
U \circ U=\left\{\begin{array}{ll}
(f, h) \in\left[L^{\infty}(\Omega, \mathcal{F}, \mu)\right]^{2} & \begin{array}{l}
\exists g \in L^{\infty}(\Omega, \mathcal{F}, \mu): \\
(f, g) \in U \text { and }(g, h) \in U
\end{array}
\end{array}\right\} .
$$

The inverse relation $U^{-1}$ of $U$ is defined by:

$$
U^{-1}=\left\{(f, g) \in\left[L^{\infty}(\Omega, \mathcal{F}, \mu)\right]^{2} \mid(g, f) \in U\right\}
$$

The diagonal $\Delta=\left\{(f, f) \in\left[L^{\infty}(\Omega, \mathcal{F}, \mu)\right]^{2}\right\}$ is the identity relation on $L^{\infty}(\Omega, \mathcal{F}, \mu)$.

Definition 5.1 A family $\mathcal{U}$ of subsets of $\left[L^{\infty}(\Omega, \mathcal{F}, \mu)\right]^{2}$ is a uniformity for $L^{\infty}(\Omega, \mathcal{F}, \mu)$ if it satisfies the following conditions.

(i) $\Delta \subset U$ for every $U \in \mathcal{U}$.

(ii) $U^{-1} \in \mathcal{U}$ for every $U \in \mathcal{U}$.

(iii) For every $U \in \mathcal{U}$ there exists $V \in \mathcal{U}$ such that $V \circ V \subset U$.

(iv) $U \cap V \in \mathcal{U}$ for every $U, V \in \mathcal{U}$.

(v) $U \in \mathcal{U}$ and $U \subset V$ imply $V \in \mathcal{U}$.

The pair $\left(L^{\infty}(\Omega, \mathcal{F}, \mu), \mathcal{U}\right)$ is a uniform space. A subfamily $\mathcal{B}$ of a uniformity $\mathcal{U}$ is a base for $\mathcal{U}$ if for every $U \in \mathcal{U}$ there exists $V \in \mathcal{B}$ such that $V \subset U$. For $U \subset\left[L^{\infty}(\Omega, \mathcal{F}, \mu)\right]^{2}$ and $f \in L^{\infty}(\Omega, \mathcal{F}, \mu)$, define $U(f)=\left\{g \in L^{\infty}(\Omega, \mathcal{F}, \mu) \mid\right.$ $(f, g) \in U\}$. The uniform topology $\tau_{\mathcal{U}}$ of $L^{\infty}(\Omega, \mathcal{F}, \mu)$ is the family of subsets of $L^{\infty}(\Omega, \mathcal{F}, \mu)$ given by:

$$
\tau_{\mathcal{U}}=\left\{O \subset L^{\infty}(\Omega, \mathcal{F}, \mu) \mid \forall f \in O \exists U \in \mathcal{U}: U(f) \subset O\right\}
$$

where the neighborhood base of $f$ is the family $\{U(f) \mid U \in \mathcal{B}\}$. Let $X$ be a subset of $L^{\infty}(\Omega, \mathcal{F}, \mu)$. The relative uniformity $\mathcal{U}_{X}$ for $X$ is the family:

$$
\mathcal{U}_{X}=\left\{U \cap(X \times X) \subset\left[L^{\infty}(\Omega, \mathcal{F}, \mu)\right]^{2} \mid U \in \mathcal{U}\right\}
$$

It induces a uniform topology $\tau_{\mathcal{U}_{X}}$ on $X$ that coincides with the topology induced by $\tau_{\mathcal{U}}$.

We present the following result from Kelley (1955, Theorem 6.26) as adapted to our context. 
Theorem 5.1 Let $\left(L^{\infty}(\Omega, \mathcal{F}, \mu), \mathcal{U}\right)$ be a uniform space and $X$ be a subset of $L^{\infty}(\Omega, \mathcal{F}, \mu)$. If $f: X \rightarrow \mathbb{R}$ is uniformly continuous, then there exists a unique uniformly continuous extension $\hat{f}: \mathrm{cl} X \rightarrow \mathbb{R}$ of $f$, where $\mathrm{cl} X$ is the closure of $X$ with respect to the uniform topology for $\left(L^{\infty}(\Omega, \mathcal{F}, \mu), \mathcal{U}\right)$.

There are many different uniformities for $L^{\infty}(\Omega, \mathcal{F}, \mu)$. Our focus here is on the uniformity for $L^{\infty}(\Omega, \mathcal{F}, \mu)$ that is consistent with the weak* topology of $L^{\infty}(\Omega, \mathcal{F}, \mu)$. The existence of such uniformity is guaranteed by the following result.

Proposition 5.1 The family $\mathcal{B}$ of all subsets $U$ of $\left[L^{\infty}(\Omega, \mathcal{F}, \mu)\right]^{2}$ given as:

$$
U=\left\{(f, g) \in\left[L^{\infty}(\Omega, \mathcal{F}, \mu)\right]^{2}|| \mu_{i}(f)-\mu_{i}(g) \mid<\varepsilon, i=1, \ldots, m\right\}
$$

for some $m \in \mathbb{N}, \mu_{1}, \ldots, \mu_{m} \in c a(\Omega, \mathcal{F}, \mu)$ and $\varepsilon>0$, is a base for a uniformity for $L^{\infty}(\Omega, \mathcal{F}, \mu)$.

Proof The family $\mathcal{B}$ is a base for a uniformity for $L^{\infty}(\Omega, \mathcal{F}, \mu)$ if and only if it satisfies the following conditions. (See Kelley 1955, Theorem 6.2.)

(i) $\Delta \subset U$ for every $U \in \mathcal{B}$.

(ii) For every $U \in \mathcal{B}$ there exists $V \in \mathcal{B}$ such that $V \subset U^{-1}$.

(iii) For every $U \in \mathcal{B}$ there exists $V \in \mathcal{B}$ such that $V \circ V \subset U$.

(iv) For every $U, V \in \mathcal{B}$ there exists $W \in \mathcal{B}$ such that $W \subset U \cap V$.

We verify that these conditions are satisfied for $\mathcal{B}$.

(i): Obvious.

(ii): This follows from the symmetry $U^{-1}=U$ for every $U \in \mathcal{B}$.

(iii): Let $U \in \mathcal{B}$ be given by:

$$
U=\left\{(f, g) \in\left[L^{\infty}(\Omega, \mathcal{F}, \mu)\right]^{2}|| \mu_{i}(f)-\mu_{i}(g) \mid<\varepsilon, i=1, \ldots, m\right\}
$$

with $m \in \mathbb{N}, \mu_{1}, \ldots, \mu_{m} \in c a(\Omega, \mathcal{F}, \mu)$ and $\varepsilon>0$. Take $V \in \mathcal{B}$ such that:

$$
V=\left\{(f, g) \in\left[L^{\infty}(\Omega, \mathcal{F}, \mu)\right]^{2}|| \mu_{i}(f)-\mu_{i}(g) \mid<\frac{\varepsilon}{2}, i=1, \ldots, m\right\} .
$$

If $(f, h) \in V \circ V$, then there exists $g \in L^{\infty}(\Omega, \mathcal{F}, \mu)$ such that $(f, g) \in V$ and $(g, h) \in$ $V$. Thus, $\left|\mu_{i}(f)-\mu_{i}(h)\right| \leq\left|\mu_{i}(f)-\mu_{i}(g)\right|+\left|\mu_{i}(g)-\mu_{i}(h)\right|<\varepsilon / 2+\varepsilon / 2=\varepsilon$ for each $i=1, \ldots, m$. This shows that $(f, h) \in U$. Hence $V \circ V \subset U$.

(iv): Let $U, V \in \mathcal{B}$ have the form:

$$
U=\left\{(f, g) \in\left[L^{\infty}(\Omega, \mathcal{F}, \mu)\right]^{2}|| \mu_{i}(f)-\mu_{i}(g) \mid<\varepsilon, i=1, \ldots, m\right\}
$$

with $m \in \mathbb{N}, \mu_{1}, \ldots, \mu_{m} \in c a(\Omega, \mathcal{F}, \mu)$ and $\varepsilon>0$, and

$$
V=\left\{(f, g) \in\left[L^{\infty}(\Omega, \mathcal{F}, \mu)\right]^{2}|| \lambda_{j}(f)-\lambda_{j}(g) \mid<\varepsilon^{\prime}, j=1, \ldots, n\right\}
$$


with $n \in \mathbb{N}, \lambda_{1}, \ldots, \lambda_{n} \in c a(\Omega, \mathcal{F}, \mu)$ and $\varepsilon^{\prime}>0$. Define the $(m+n)$-dimensional vector measure by $\vec{\theta}=\left(\mu_{1}, \ldots, \mu_{m}, \lambda_{1}, \ldots, \lambda_{n}\right)$. Then, the intersection $U \cap V$ contains the set $W \in \mathcal{B}$ given by:

$$
W=\left\{\begin{array}{ll}
(f, g) \in\left[L^{\infty}(\Omega, \mathcal{F}, \mu)\right]^{2} & \begin{array}{l}
\left|\theta_{k}(f)-\theta_{k}(g)\right|<\min \left\{\varepsilon, \varepsilon^{\prime}\right\} \\
k=1, \ldots, m+n
\end{array}
\end{array}\right\},
$$

where $\theta_{k}$ are component measures of $\vec{\theta}$.

Let $\left(L^{\infty}(\Omega, \mathcal{F}, \mu), \mathcal{U}\right)$ be the uniform space with the base $\mathcal{B}$ for $\mathcal{U}$ given in Proposition 5.1. By construction, the sets of the form:

$$
U(f)=\left\{g \in L^{\infty}(\Omega, \mathcal{F}, \mu)|| \mu_{i}(f)-\mu_{i}(g) \mid<\varepsilon, i=1, \ldots, m\right\}
$$

with $m \in \mathbb{N}, \mu_{1}, \ldots, \mu_{m} \in c a(\Omega, \mathcal{F}, \mu)$ and $\varepsilon>0$, constitute a neighborhood base of $f$ for the weak* topology of $L^{\infty}(\Omega, \mathcal{F}, \mu)$. (See Subsection 2.2.) Therefore, the uniform topology and the weak* topology coincide and the relative uniform topology of $X \subset L^{\infty}(\Omega, \mathcal{F}, \mu)$ coincides with the relative weak* topology of $X$.

\subsection{The structure of the Pareto frontier}

The next lemma is an immediate consequence of the Banach-Alaoglu theorem (see Dunford and Schwartz 1958, Corollary V.4.3).

Lemma 5.1 $\mathcal{A}$ is weakly* compact in $\left[L^{\infty}(\Omega, \mathcal{F}, \mu)\right]^{n}$.

Lemma 5.2 Suppose that $v_{i}$ is uniformly continuous and strictly $\mu_{i}$-monotone for each $i=1, \ldots, n$. Then, an allocation is Pareto optimal if and only if it is weakly Pareto optimal.

Proof It is evident that Pareto optimality implies weak Pareto optimality. We show the converse implication. Let $\left(f_{1}, \ldots, f_{n}\right)$ be an allocation for $\widehat{\mathcal{E}}$ that is not Pareto optimal. Then, there is an allocation $\left(g_{1}, \ldots, g_{n}\right)$ in $\mathcal{A}$ such that $\hat{v}_{i}\left(f_{i}\right) \leq \hat{v}_{i}\left(g_{i}\right)$ for each $i$ and $\hat{v}_{j}\left(f_{j}\right)<\hat{v}_{j}\left(g_{j}\right)$ for some $j$. As $\hat{v}_{j}$ is strictly $\mu_{j}$-monotone by Theorem 2.2, there exists $A \in \mathcal{F}$ with $\mu(A)>0$ on which $g_{j}$ is positive. The mutual absolute continuity of $\mu_{1}, \ldots, \mu_{n}$ yields $\mu_{i}(A)>0$ for each $i$. By the weak* continuity of $\hat{v}_{j}$ established in Theorem 2.1, there is $\varepsilon \in(0,1)$ such that $\hat{v}_{j}\left(f_{j}\right)<\hat{v}_{j}\left((1-\varepsilon) g_{j}\right)$. Define $h_{i} \in L^{\infty}(\Omega, \mathcal{F}, \mu)$ by

$$
h_{i}= \begin{cases}g_{i}+\frac{\varepsilon}{n-1} g_{j} & \text { if } i \neq j \\ (1-\varepsilon) g_{j} & \text { otherwise }\end{cases}
$$

It is easy to see that $0 \leq h_{i} \leq 1$ for each $i, h_{i} \geq g_{i}$ and $\mu_{i}\left(h_{i}\right)=\mu_{i}\left(g_{i}\right)+\varepsilon \mu_{i}\left(g_{j}\right) /$ $(n-1)>\mu_{i}\left(g_{i}\right)$ for $i \neq j$. By the strict $\mu_{i}$-monotonicity of $\hat{v}_{i}$ established in Theorem 2.2, the resulting allocation $\left(h_{1}, \ldots, h_{n}\right)$ satisfies $\hat{v}_{i}\left(f_{i}\right)<\hat{v}_{i}\left(h_{i}\right)$ for each $i$. Thus, allocation $\left(f_{1}, \ldots, f_{n}\right)$ is not weakly Pareto optimal. 
For every Pareto optimal allocation in $\widehat{\mathcal{E}}$, an individual exists that no one envies. This is a variant of the simple observation by Varian (1974), which plays an important role in proving the existence of a Pareto optimal envy-free allocation.

Proposition 5.1 For every Pareto optimal allocation $\left(f_{1}, \ldots, f_{n}\right)$ there exists $j$ such that $\hat{v}_{i}\left(f_{j}\right) \leq \hat{v}_{i}\left(f_{i}\right)$ for each $i=1, \ldots, n$.

Proof Take an arbitrary Pareto optimal allocation $\left(f_{1}, \ldots, f_{n}\right)$. Suppose, to the contrary, that for each $j$ there exists $\pi(j) \in\{1, \ldots, n\}$ such that $\hat{v}_{\pi(j)}\left(f_{\pi(j)}\right)<\hat{v}_{\pi(j)}\left(f_{j}\right)$. Then, the map $\pi$ from $\{1, \ldots, n\}$ into itself defined by $j \mapsto \pi(j)$ satisfies $\pi(j) \neq j$ for each $j$. Thus, we have $\pi^{s}(j) \neq \pi^{s+1}(j)$ and $\hat{v}_{\pi^{s+1}(j)}\left(f_{\pi^{s+1}(j)}\right)<\hat{v}_{\pi^{s+1}(j)}\left(f_{\pi^{s}(j)}\right)$ for every $s=0,1, \ldots$, where $\pi^{s}$ is the $s$-th iteration of $\pi$ with $\pi^{0}$ the identity map on $\{1, \ldots, n\}$. As the sequence $\left\{\pi^{s}(j)\right\}_{s=0}^{\infty}$ is contained in $\{1, \ldots, n\}$, and hence finite, we have $\pi^{s}(j)=\pi(j)^{s-t}$ for some integers $s>t \geq 0$. Let $i_{0}=\pi^{s}(j), i_{1}=\pi^{s-1}(j), \ldots, i_{t}=\pi^{s-t}(j)$ and $I=\left\{i_{0}, \ldots, i_{t}\right\}$. It is evident that $\hat{v}_{i_{0}}\left(f_{i_{0}}\right)<\hat{v}_{i_{0}}\left(f_{i_{1}}\right), \ldots, \hat{v}_{i_{t-1}}\left(f_{i_{t-1}}\right)<\hat{v}_{i_{t-1}}\left(f_{i_{t}}\right)$, and $\hat{v}_{i_{t}}\left(f_{i_{t}}\right)<\hat{v}_{i_{t}}\left(f_{i_{0}}\right)$. Define the allocation $\left(g_{1}, \ldots, g_{n}\right)$ by:

$$
g_{i}= \begin{cases}f_{i_{k+1}} & \text { if } i=i_{k} \text { for } 1 \leq k \leq t-1, \\ f_{i_{0}} & \text { if } i=i_{t} \\ f_{i} & \text { if } i \notin I\end{cases}
$$

It is obvious that the resulting allocation $\left(g_{1}, \ldots, g_{n}\right)$ satisfies $\hat{v}_{i}\left(f_{i}\right)<\hat{v}_{i}\left(g_{i}\right)$ for each $i \in I$ and $\hat{v}_{i}\left(g_{i}\right)=\hat{v}_{i}\left(f_{i}\right)$ for each $i \notin I$. This contradicts the Pareto optimality of $\left(f_{1}, \ldots, f_{n}\right)$.

The weak* continuity of $\hat{v}_{i}$ by Theorem 2.1 and the weak* compactness of $\mathcal{A}$ by Lemma 5.1 guarantee that $\Gamma$ is compact in $\mathbb{R}^{n}$ and that $\Gamma^{\mathrm{P}}$ is nonempty and closed by Lemma 5.2. It follows from the strict $\mu_{i}$-monotonicity of $\hat{v}_{i}$ that $\Gamma^{\mathrm{P}}$ is included in the boundary of $\Gamma$. Note also that $\Gamma$ is comprehensive from below. That is, $\left(x_{1}, \ldots, x_{n}\right) \in \Gamma$ and $0 \leq\left(y_{1}, \ldots, y_{n}\right) \leq\left(x_{1}, \ldots, x_{n}\right)$ imply $\left(y_{1}, \ldots, y_{n}\right) \in \Gamma$.

Under our hypotheses, the Pareto frontier is homeomorphic to the unit simplex. The following technique to demonstrate this significant property is based on the argument developed by Hüsseinov (2009), Mas-Colell (1986), Sagara (2008).

Proposition 5.2 Define the function $\rho: \Delta^{n-1} \rightarrow \mathbb{R}$ by

$$
\rho(x)=\sup \{r \geq 0 \mid r x \in \Gamma\},
$$

and let $h: \Delta^{n-1} \rightarrow \mathbb{R}^{n}$ be defined by:

$$
h(x)=\rho(x) x .
$$

Then, $h$ is a homeomorphism between $\Delta^{n-1}$ and $\Gamma^{\mathrm{P}}$.

Proof It follows from the closedness of $\Gamma$ that $h(x) \in \Gamma$. If $h(x) \notin \Gamma^{\mathrm{P}}$, then there exists $y \in \Gamma$ such that $h(x)<y$. This implies that $0 \leq(\rho(x)+\varepsilon) x<y$ for any 
sufficiently small $\varepsilon>0$, and hence $(\rho(x)+\varepsilon) x \in \Gamma$. This contradicts the definition of $\rho$. Therefore, $h$ is a mapping from $\Delta^{n-1}$ into the compact set $\Gamma^{\mathrm{P}}$. By the strict $\mu_{i}$-monotonicity of $\hat{v}_{i}$, it is evident that $\Gamma$ contains a strictly positive vector. Hence, $\rho(x)>0$ for every $x \in \Delta^{n-1}$ because $\Gamma$ is comprehensive from below. It follows easily from this that $h$ is an injection.

We show that $h: \Delta^{n-1} \rightarrow \Gamma^{\mathrm{P}}$ is a surjection. To this end, choose any $y \in \Gamma^{\mathrm{P}}$. Note that $y$ is nonzero by the strict $\mu_{i}$-monotonicity of $v_{i}$. Define $x_{i}=y_{i} / \sum_{k=1}^{n} y_{k}$ for each $i$. Then, we have $x \in \Delta^{n-1}$ and $y=\sum_{k=1}^{n} y_{k} x$. Suppose that $\sum_{k=1}^{n} y_{k} \neq \rho(x)$. By the definition of $\rho(x)$ and the fact that $\sum_{k=1}^{n} y_{k} x \in \Gamma^{\mathrm{P}}$, we must have $\sum_{k=1}^{n} y_{k}<\rho(x)$. Thus, $y_{i}=\sum_{k=1}^{n} y_{k} x_{i} \leq \rho(x) x_{i}$ for each $i$ and $y_{j}=\sum_{k=1}^{n} y_{k} x_{j}<\rho(x) x_{j}$ for some $j$ with $x_{j}>0$. This contradicts the fact that $y \in \Gamma^{\mathrm{P}}$ in view of $h(x)=\rho(x) x \in \Gamma^{\mathrm{P}}$. Thus we have $\sum_{k=1}^{n} y_{k}=\rho(x)$, and hence $h(x)=y$.

Since $\Delta^{n-1}$ is compact, to complete the proof it suffices to show that $h$ is continuous. This will follow if we show that $\rho$ is a continuous function. To show the upper semicontinuity of $\rho$, assume, by way of contradiction, that $x^{k} \in \Delta^{n-1}$ and $x^{k} \rightarrow x$ imply $\overline{\lim }_{k} \rho\left(x^{k}\right)>\rho(x)$. Then, as $\rho$ is bounded, there exists a subsequence $\left\{x^{k_{m}}\right\}$ of sequence $\left\{x^{k}\right\}$ such that $\rho\left(x^{k_{m}}\right) \rightarrow r_{0}>\rho(x)$. The closedness of $U$ implies $r_{0} x \in \Gamma$. But, $r_{0}>\rho(x)$ contradicts the definition of $\rho$. To demonstrate the lower semicontinuity of $\rho$, assume, by way of contradiction, that $x^{k} \in \Delta^{n-1}$ and $x^{k} \rightarrow x$ imply $\underline{\lim }_{k} \rho\left(x^{k}\right)<\rho(x)$. Then, there exists a subsequence $\left\{x^{k_{m}^{\prime}}\right\}$ of $\left\{x^{k}\right\}$ such that $\rho\left(x^{k_{m}^{\prime}}\right) \rightarrow r_{0}^{\prime}<\rho(x)$. Thus, $\left\{\rho\left(x^{k_{m}^{\prime}}\right) x^{k_{m}^{\prime}}\right\}$ is a sequence in $\Gamma^{\mathrm{P}}$ with the limit $r_{0}^{\prime} x$ not in $\Gamma^{\mathrm{P}}$. This contradicts the closedness of $\Gamma$.

\section{References}

Akin E (1995) Vilfred Pareto cuts the cake. J Math Econ 24:23-44

Aumann RJ, Shapley LS (1974) Values of non-atomic games. Princeton University Press, Princeton

Barbanel JB (2005) The geometry of efficient fair division. Cambridge University Press, Cambridge

Berliant M, Thomson W, Dunz K (1992) On the fair division of a heterogeneous commodity. J Math Econ 21:201-216

Dall'Aglio M, Maccheroni F (2009) Disputed lands. Games Econ Behav 66:57-77

Dubins LE, Spanier EH (1961) How to cut a cake fairly. Am Math Mon 68:1-17

Dunford N, Schwartz JT (1958) Linear operators, part I: general theory. Wiley, New York

Dvoretsky A, Wald A, Wolfowitz J (1951) Relations among certain ranges of vector measures. Pac J Math 1:59-74

Edwards DA (1987) On a theorem of Dvoretsky, Wald, and Wolfowitz concerning Liapounov measures. Glasg Math J 29:205-220

Einy E, Moreno D, Shitovitz B (1999) The core of a class of non-atomic games which arise in economic applications. Int J Game Theory 28:1-14

Gale D, Mas-Colell A (1975) An equilibrium existence theorem for a general model without ordered preferences. J Math Econ 2:9-16; Corrections, (1979) ibid. 6:297-298

Hüsseinov F (2009) $\alpha$-Maximin solutions to fair division problems and the structure of the set of Pareto utility profiles. Math Soc Sci 57:279-281

Hüsseinov F (2011) A theory of a heterogeneous divisible commodity exchange economy. J Math Econ 48:54-59

Hüsseinov F, Sagara N (2012) Concave measures and the fuzzy core in exchange economies with heterogeneous divisible commodities. Fuzzy Sets Syst 198:70-82

Kelley JL (1955) General topology. Van Nostrand, Princeton

Khan MA, Rath KP (2009) On games with incomplete information and the Dvoretsky-Wald-Wolfowitz theorem with countable partitions. J Math Econ 45:830-837 
Kingman JFC, Robertson AP (1968) On a theorem of Lyapunov. J Lond Math Soc 43:347-351

Lindenstrauss J (1966) A short proof of Lyapunov's convexity theorem. J Math Mech 15:971-972

Lyapunov A (1940) Sur les fonctions-vecteurs complétement additives. Bull Acad Sci URSS Sér Math 4:465-478

Mas-Colell A (1986) The price equilibrium existence problem in topological vector lattices. Econometrica 54:1039-1053

Rudin W (1991) Functional analysis, 2nd edn. McGraw-Hill, New York

Sagara N (2008) A characterization of $\alpha$-maximin solutions of fair division problems. Math Soc Sci 55:273280

Sagara N, Vlach M (2010) Convexity of the lower partition range of a concave vector measure. Adv Math Econ 13:155-160

Sagara N, Vlach M (2011) A new class of convex games and the optimal partitioning of measurable spaces. Int J Game Theory 40:617-630

Scarf H (1967) The approximation of fixed points of a continuous mapping. SIAM J Appl Math 15:13281343

Scarf H (1973) The computation of economic equilibria. Yale University Press, New Haven

Steinhaus H (1948) The problem of fair division. Econometrica 16:101-104

Varian HR (1974) Equity, envy, and efficiency. J Econ Theory 9:63-91

Weller D (1985) Fair division of a measurable space. J Math Econ 14:5-17 